\title{
Quality of Service (QoS) for 5G Networks
}

\author{
Raed Abduljabbar Aljiznawi, Naseer Hwaidi Alkhazaali, Saba Qasim Jabbar, and Dheyaa Jasim \\ Kadhim
}

\begin{abstract}
This work explores some important number of key performance indicators (KPIs) that may affect on the quality of service $(Q \circ S)$ in $5 G$ networks. The proposed QoS necessities are based on the analysis of functional requirements to $5 \mathrm{G}$ networks and traffic parameters for $\mathrm{HD}$ video and massive M2M services, which will be highly demanded in 2020 . One of the 5G development paradigms is the network functions virtualization (NFV) including cloud radio access and cloud core networks. This work has planned the concept of function blocks; cloud QoS management function (CQMF) and cloud QoS control function (CQCF) to control and monitor QoS, which is implemented as part of the $5 \mathrm{G}$ network cloud infrastructure.
\end{abstract}

Index Terms-5G, M2M, QoS, video services, virtualization.

\section{INTRODUCTION}

The new generation of mobile networks will be amplified in the future, will be expected to facilitate connections with a data rates that are greater than current mobile networks with 1000 times more data by volume in any location and up to 100 times as many connected devices [1]. However, in order to meet the needy Key Performance Indicators (KPIs) [2], [3] set for $5 \mathrm{G}$ networks, to overcome a number of important problems researchers will need to conduct. Minimum of the problems will be that of supervision the expected exponential growth in multimedia traffic and meet the quality expectations of end users. Irrespective to that the predicted massive increase in numbers of devices attached to $5 \mathrm{G}$ networks, the 5G KPIs expect that Ultra High-Definition (UHD) video [4] will be facilitated via a range of applications such as Internet Protocol Television (IPTV) and Video on Demand services (VoD) with each UHD stream potentially requiring up to 16 times as much bandwidth as current High-Definition (HD) streams.

According to latest networking forecast [5], mobile video will account for about $75 \%$ of all mobile data traffic by 2020 , representing a 13-fold increase since 2012. Software-Defined Networking (SDN) [6] has been generally playing an important role in 5G networks. In SDN, the control plane is connected from the data (forwarding) plane. This disjointing has the capability to allow an SDN controller to catch an overview of not only the entire network containing up to date Quality of Service (QoS) information on all paths in the network but also an overview of all competing for traffic

Manuscript received April 22, 2016; revised January 23, 2017.

A. Raed, H. Naseer, and Q. Saba are with Electronics and Information Engineering School, Huazhong University and Science and Technology (HUST), Wuhan, China (e-mail: raeid72falih@yahoo.com, naserstar2007@yahoo.com, shura2007515@yahoo.com).

J. Dheyaa is with the Electrical Engineering Department, University of Baghdad, Baghdad (e-mail: dheyaa@coeng.uobaghdad.edu.iq). flows at present reversing the network. Having access to both views could provide great potential advantages when managing the transit of bandwidth-hungry, delay-intolerant multimedia flows over the $5 \mathrm{G}$ network. However, SDN is still a current research area where ways to the quality of service $(\mathrm{QoS})$ provisioning and traffic engineering for multimedia applications have yet to reach maturity. Standardization in 2013 of High-Efficiency Video Coding (HEVC) [7] as H.265 by the ITU-T offers a newer alternative that improves compression ratios (over H.264/AVC) by up to $40 \%$ with no loss in quality. In 2012, a second version of the H.265 standard added a scalable extension [8], which also delivers very substantial bandwidth savings over H.264/SVC.

Mobile networks have diligence with the trend of becoming more diverse due to the addition of more upright markets or services like healthcare and Machine-to-Machine (M2M) niches integrating with data networks [9]. Researchers in both industry and the academic world keep on to face the challenge of proficiently streaming high volumes of high-quality video with guaranteed end-to-end QoS, and high quality of experience (QoE) for the end user because of this trend.

The rest of this paper is organized as follows: Section 2 will describe the $5 \mathrm{G}$ networks technological infrastructure, while section 3 gives the implementation of SDN-based 5G networks architecture. Section 4 gives the conceptive description about the traffic at $5 \mathrm{G}$ networks. Section 5 describes the QoS management for $5 \mathrm{G}$ networks, and finally Section 6 gives the important conclusions are drawn from this work.

\section{5G Networks TeChNOLOGICAL INFRASTRUCtURE}

Technological growth of $5 \mathrm{G}$ networks will be aimed at the creation of ultra-dense networks (UDN) of wireless access with assorted cells arrangement and radius of not more than 50 meters. 5G Networks will be based on new methods of modulation and transmission that will significantly increase the spectral efficiency compared with $4 \mathrm{G}$ networks and ensure data transfer speed of more than $10 \mathrm{~Gb} / \mathrm{s}$. To provide such data transfer speed in $5 \mathrm{G}$ networks the use of broadband channels in the downlink (DL), as well as in the uplink (UL) with a continuous spectrum width of 500 to $1000 \mathrm{MHz}$ will be required. This amount of spectrum is $25-50$ times wider than the channels width used in 4G. Allocation of these bands for $5 \mathrm{G}$ channels is possible only at the upper boundary of the centimeter and in millimeter wave bands that will significantly reduce base stations coverage up to $50-100 \mathrm{~m}$ [10]. The increase in spectral efficiency of 5G networks can be achieved using non-orthogonal multiple access methods 
(NOMA) in RAN networks and using non-orthogonal signals (e.g., FTN-signals, F-OFDM-signals, etc.) [11]. Requirements to the cell's spectral efficiency in $5 \mathrm{G}$ networks for divergent transmission channels are very similar to these requirements with $4 \mathrm{G}$ networks shows the growth of spectral efficiency by 3-5 times.

$5 \mathrm{G}$ network infrastructure will be based on the use of cloud technologies, both in radio access networks (Cloud RAN) with using Software Defined Radio (SDR) infrastructure and in the core network (Cloud CN) with using Software Defined Network (SDN) infrastructure. Full virtualization of NFV network functions implemented in $5 \mathrm{G}$ infrastructure will take place. This virtualization of NFV network functions should cover the control and management of QoS, the service policy and prioritization of traffic [12]. New solutions for 5G networks will be the appearance of moving $5 \mathrm{G}$ nodes (base stations) and moving $5 \mathrm{G}$ backhaul that is dictated by the need to implement $5 \mathrm{G}$ during the construction of intelligent transport network. Thanks to these solutions, international highways with cars moving at a speed exceeding $200 \mathrm{~km} / \mathrm{h}$ will introduce moving $5 \mathrm{G}$ communication networks constructed on the basis of M2M applications and devices for Vehicle-to-Vehicle (V2V) scenario and ensuring the safety traffic and multimedia data exchange. The role of $5 \mathrm{G}$ base stations will carry out the $5 \mathrm{G}$ vehicle devices united in a mesh network.

\section{IMPLEMENTATION OF SDN FOR 5G NETWORKS}

Software defined networking (SDN) is bringing about a paradigm shift in networking through the ideas of programmable network infrastructure and decoupling of network control and data planes. It promises simplified network management and easier introduction of new services or changes into the network. Use of SDN concepts in 4G/5G mobile cellular networks is also being seen to be beneficial (e.g., for more effective radio resource allocation through centralization, seamless mobility across diverse technologies through a common control plane).

$5 \mathrm{G}$ networks is going to be designed to be open, more flexible, and able to evolve more easily than the traditional networks, and will not be based on routing and switching technologies anymore [4]. It is able to provide convergent network communication across multi-technologies networks, and provide open communication system to cooperate with satellite systems, cellular networks, clouds and data-centers, home gateways, and many more open networks and devices. Additionally, 5G systems will be autonomous and sufficiently able to adapt the situations depending in required QoS to handle application-driven networks dynamically. Security, resiliency, robustness and data integrity will be the first priority in the design of future networks [5]. Moreover, the $5 \mathrm{G}$ network will be able of handling user-mobility to guarantee connectivity at any situation. The end users' terminals make the final choice among different access networks for the best connection. Also, the terminals will also stay awake and looking for to choose the best technology to connect, with respect to the dynamic changes at the current access technology. The infrastructure of the wireless networks will be based on SDN, which provides arrange the communication between the applications and services in the cloud and user's mobile terminal. Therefore, the network can be managed on the real-time needs and status dynamically, and it will have benefit from resource virtualization. The architecture of $5 \mathrm{G}$ network based on SDN scheme has shown in Fig. 1 below.

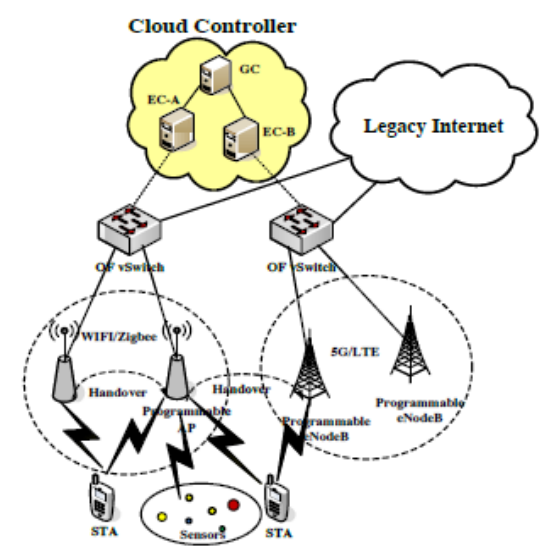

Fig. 1. SDN based $5 \mathrm{G}$ oriented network architecture.

SDN has several limitations, it has some advantages such as resource sharing and session management. The main limitation is on the computing capabilities and resources of mobile devices. As a result because mobile users send request over and over to the embedded controller for flow rules in Open Flow messages, the overhead increases more significantly. Hence, further researches are required on leveraging SDN for $5 \mathrm{G}$ networks.

\section{TRAFFIC IN 5G NETWORKS}

When forming necessities to QoS in 5G networks two key traffic models should be firstly considered: high-speed video flow "server-subscriber" and massive M2M. Video transmission services will be a significant stimulus to the development and a rapidly mounting segment of $5 \mathrm{G}$ networks traffic. In 2014, the volume of video services in the total traffic of $4 \mathrm{G}$ networks subscribers already exceeded $50 \%$, and by 2020 , it is forecasted to increase at least by 13 times [13]. Thus, we can already observe the first wave of incoming "tsunami" of subscribers' traffic in $4 \mathrm{G}$ networks. Monthly use of data transmission traffic in $4 \mathrm{G}$ networks has already reached $2.6 \mathrm{~GB}$ and a monthly consumption of traffic in $5 \mathrm{G}$ networks will exceed $500 \mathrm{~GB}$. The growth of video services traffic volume will be associated with the implementation of various technologies of video services image quality from standard SD TV to UHD TV (8k), which in its turn requires a data transmission speed of up to $10 \mathrm{~Gb} / \mathrm{s}$ in the network.

The development of NFV concept will lead to virtualization of distinction management function that could be introduced in the form of two main functions: Cloud QoS management function (CQMF) and Cloud QoS control function (CQCF) shown in Fig. 2 below.

CQCF function of QoS control supplies real-time control of traffic flows in 5G network on the basis of QoS levels recognized during the connection. Basic QoS control mechanisms include traffic profiling, planning, and management of data flows. CQMF function of QoS 
management provides QoS support in 5G network in accordance with SLA service contracts, as well as supplies monitoring, maintenance, review and scaling of QoS. Implementation of algorithms for traffic prioritization in $5 \mathrm{G}$ networks will be based on traffic classification procedures with a focus on video traffic priorities and M2M traffic [14].

Traffic classification procedure should be done taking into deliberation the adaptation possibility, as the traffic characteristics will vigorously change with the emergence of new applications, both in M2M area and in the field of video services.

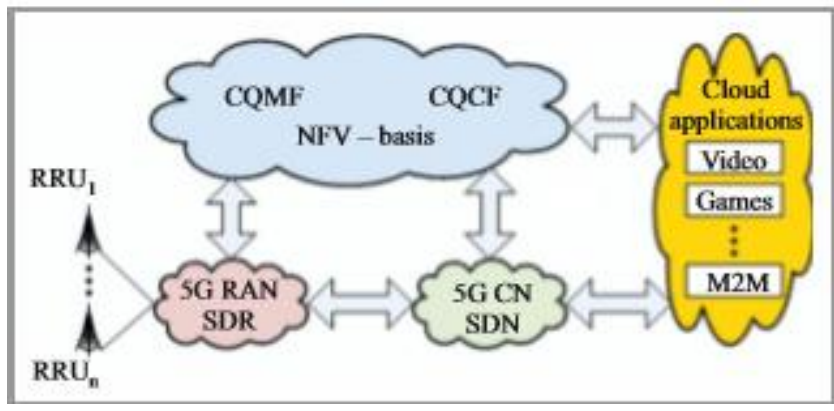

Fig. 2. Virtualization of control and management functions in $5 \mathrm{G}$ network.

Whenever blockage is detected on a primary path transport function flows that are part of a stream managed by a video quality assurance manager (VQAM), it re-evaluates the QoE of the primary and secondary paths and performs one of the following five possible actions: Make secondary path primary path; Switch only improvement layers to secondary path; Delete flows containing augmentation layers (starting with the highest layer) until QoE utility of stream meets minimum QoE service level threshold; Perform a new extensive path search and recalculate QoE utility for all available paths selecting new primary and secondary paths; Report malfunction to meet minimum QoE service level threshold to SDN-VQO. The VQAM decision-making process firstly determines if the secondary path can fulfill the required QoE utility function and if so simply informs the SDN Controller to switch all flows belonging to the multimedia stream to use the secondary path. Where neither the primary nor the secondary path can individually meet the QoE service level, a concurrent multipath transmission is employed to cumulative the flows over both primary and secondary paths. In this case, the base layer will be sent over the path offering the highest QoE utility and the development layers over the alternate path [15]. Should extra congestion occur, the VQAM may alleviate this by selectively dropping scalable layers from a multimedia stream by instructing the SDN controller to remove flow information from SDN forwarder.

In the case where neither of the primary or the secondary paths can successfully deliver the base layer, the VQAM will initiate a reset situation for the affected video stream and seek to find a new set of paths capable of meeting the QoE utility threshold. In the event that the VQAM is unable to provide the mandatory level of utility, it will report this failure to the SDN-VQO, which can then take global action to alleviate the network congestion [16].

The 5G mobile telecommunication average has been conceived for a while now as a successor to the current $4 \mathrm{G}$ cellular standards to address the forecasted traffic volume growth, and the inflexibility and difficulties in innovation plaguing the hardware-based architectures at the front end and core of these networks. In count to these, 5G standard proposes better or advanced performance and societal KPIs. Detailed images of some of the KPIs in $5 \mathrm{G}$ are discussed in [2].

The main KPIs identified include:

1) Traffic volume density

2) Experienced end user throughput

3) Latency

4) Reliability

5) Availability and retain ability

\section{QOS MANAGEMENT IN 5G NeTWORKS}

During the evolution of QoS management device in 3GPP (GSM/UMTS/LTE) networks, there was a migration from QoS management at the user equipment level to the QoS management at the network level. This approach to QoS management will be maintained in 5G networks as well. QoS management mechanisms in $5 \mathrm{G}$ networks should provide video and VoIP traffic prioritization towards Web search traffic and other applications tolerant to quality. The service of streaming video transfer without buffering is very sensitive to network delay, so one of the most imperative parameters that determine QoS requirements is the total packet delay budget (PDB), which is formed on the RAN air interface, and is treated as the maximum packet delay with a condensed level of $98 \%$.

Table I lists the necessities for the delay in $3 \mathrm{G} / 4 \mathrm{G} / 5 \mathrm{G}$ networks formed in 3GPP [17] and METIS project [18]. These data demonstrate that with the increase in mobile network's generation the requirements for the lower boundary of the total data delay across the network decline. Also, the analysis of the necessities for the overall $5 \mathrm{G}$ network delay revealed that given the accumulation effect the delay in 5G RAN network should be less than $1 \mathrm{~ms}$.

TABLE I: REQUIREMENTS FOR DELAY IN 3G/4G/5G NETWORKS

\begin{tabular}{|c|c|c|c|}
\hline \multirow{2}{*}{ QoS terms } & \multicolumn{3}{|c|}{ Packet delay budget [ms] } \\
\cline { 2 - 4 } & $3 \mathrm{G}$ & $4 \mathrm{G}$ & $5 \mathrm{G}$ \\
\hline \hline Without quality assurance & $\begin{array}{c}\text { Not } \\
\text { determined }\end{array}$ & $100-300$ & $\begin{array}{c}\text { Not } \\
\text { determined }\end{array}$ \\
\hline With guaranteed quality & $100-280$ & $50-300$ & 1 \\
\hline
\end{tabular}

Comparison of requirements to delay in control and user planes for signaling traffic and user traffic respectively, presented in Fig. 3 below, show that requirements to $5 \mathrm{G}$ networks will be twice more rigid for traffic in the user plane and 10 times more rigid - in the subscriber traffic plane [6]. Another parameter is the proportion of packets lost due to errors when receiving data packets (IP Packet Error Rate). Values for this parameter, that determines requirements for the largest number of IP packets lost for video broad-casting through 3G/4G/5G mobile networks.

For M2M services the quality also will be determined by the proportion of packets lost when receiving in $3 \mathrm{G} / 4 \mathrm{G} / 5 \mathrm{G}$ networks. Given that service conditions of M2M subscriber devices will be determined for both cases: with a guaranteed 
quality of service and without guarantees, requirements to the share of lost packets differ by three orders.

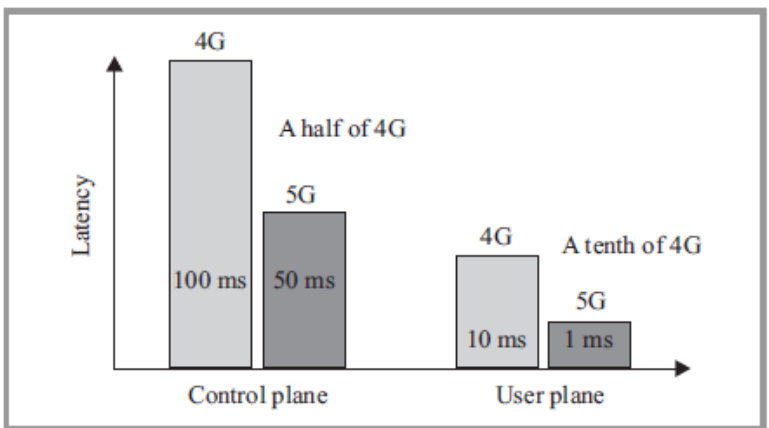

Fig. 3. Requirements to delay in control and user planes for $4 \mathrm{G} / 5 \mathrm{G}$ networks.

\section{CONCLUSIONS}

The emergence of 5G networks in the market in 2020 will be focused on a significant improvement of characteristics of mobile networks, including quality of service. Given that the principles of QoS control will be maintained during the transition from $4 \mathrm{G}$ to $5 \mathrm{G}$, the main effort of $5 \mathrm{G}$ developers should be focused on the virtualization of network functions, responsible for the management and control of QoS in the network. Another direction for development will be algorithms for traffic classification that will support market's changes including change of required for services and needs of customers. Future mobile services will be grouped around video services and services based on the massive use of M2M devices in most industries and consumers' households.

\section{REFERENCES}

[1] J. Andrews, S. Buzzi, W. Choi, S. Hanly, A. Lozano, A. C. K. Soong, and J. Zhang, "What will 5G be?" IEEE Journal on Selected Areas in Communications, vol. 32, no. 6, pp. 1065-1082, June 2014.

[2] K. Kusuma et al. (Apr 2015). Updated scenarios, requirements and KPIs for $5 \mathrm{G}$ mobile and wireless system with recommendations for future investigations. Mobile and Wireless Communications Enablers for the Twenty-Twenty Information Society (METIS) Deliverable, ICT-317669METIS/D1.5. [Online]. Available: https://www.metis2020.com/wpcontent/uploads/deliverables/METIS_ D1.5_v1.pdf

[3] 5G-PPP. Key Performance Indicators. [Online]. Available: https://5gppp.eu/kpis/

[4] E. Nakasu, "Super hi-vision on the horizon: A future tv system that conveys an enhanced sense of reality and presence," IEEE Consumer Electronics Magazine, vol. 1, no. 2, pp. 36-42, Apr 2012.

[5] Cisco. (May 2015). The zettabyte era- trends and analysis. Cisco Visual Networking Index (VNI). [Online]. Available: http://www.cisc8o.com/c/en/us/solutions/collateral/serviceprovider/vi sual-networking-indexvni/VNI_Hyperconnectivity_WP.html

[6] B. Nunes, M. Mendonca, X. Nguyen, K. Obraczka, and T. Tutti, "A survey of software-defined networking: the past, present, and future of programmable networks," IEEE Communications Surveys \& Tutorials, vol .16, no. 3, pp. 1617-1634, Aug 2014.

[7] ITU-T, "High-efficiency video coding," Rec H. 265, ver 2, Oct. 2014

[8] V. O. Tikhvinskiy, "5G world summit - 2014. Same course from 4G to 5G," Electrosvyaz, no. 7, pp. 2-39, 2014.

[9] G. Sullivan et al., "Overview of the high-efficiency video coding (HEVC) standard," IEEE Transactions on Circuits and Systems for Video Technology, vol. 22, no. 12, pp. 1649-1668, 2012.
[10] G. Bochechka and V. Tikhvinskiy, "Spectrum occupation and perspectives millimeter band utilization for 5G networks," in Proc. ITU-T Conf., Russia, 2014.

[11] J. Chimeh, "5G mobile communications: A mandatory wireless infrastructure for big data," in Proc. International Conference on Advances in Computing, Electronics and Electrical Technology (CEET), 2015, 2015.

[12] G. Wunder, "5th generation non-orthogonal waveforms for asynchronous signalling," COST Meeting 2014, Ferrara Italy, 2014.

[13] G. Bochechka and V. Tikhvinskiy, "Spectrum occupation and perspectives millimeter band utilization for 5G networks," in Proc. ITU-T Conf. "Kaleydoscope 2014, Russia, 2014.

[14] Project METIS Deliverable D2.1 Requirements and General Design Principles for New Air Interface, 2013

[15] T. Weingand, G. Sullivan, G. Bjontegaard, and A. Luthra, "Overview of the H.264/AVC video coding standard," IEEE Transactions on Circuits and Systems for Video Technology, vol. 13, no. 7, pp. 560-576, July 2003

[16] Project METIS Deliverable D2.1 Requirements and General Design Principles for New Air Interface, 31.08.2013.

[17] V. O. Tikhvinskiy, G. S. Bochechka, and A. V. Minov, "LTE network monetization based on M2M services," Electrosvyaz, no. 6, pp. 12-17, 2014.

[18] ETSI Technical Specification. Digital Video Broadcasting (DVB), Transport of MPEG-2 TS Based DVB Services over IP Based Networks, ETSI TS 102034 V1.4.1, 2009.

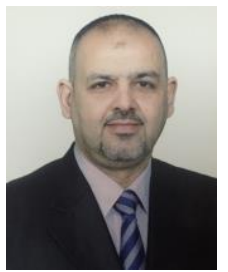

Raed A. Falih received BSc in Computer Techniques Engineering Department at University College of Madenat Al-Elem. Now he is studying the master degree electronic information and communication engineering at Huazhong University of Science and Technology in China. He works in ministry of communication in Iraq, member of the Iraqi engineers Association. He worked in the field of computer networks in Cisco Academy. He worked as assistance director in Iraqi governmental mail system project.

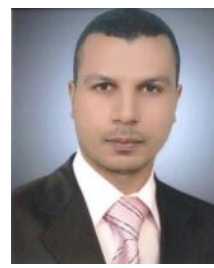

Naseer H. Abed received BSc in in communication technology at Technical College, Najaf in Iraq. He is currently a master student in communication and information engineering at Huazhong University of Science and Technology in China. He gets some certificates in networking such as Cisco and Huawei He has some international published papers in journals and conf. He works in ministry of communication in Iraq. Her field of interests is wireless networks, fiber optical communication and mobile communication systems.

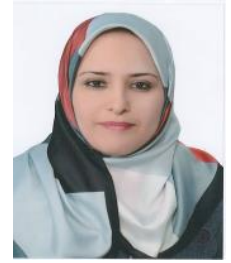

Saba Q. Jabbar received BSc in computer engineering at Baghdad University; the MSc in communication and information engineering at Huazhong University of Science and Technology in China. She is currently a $\mathrm{PhD}$ student at Huazhong University of Science and Technology in China. She works as a faculty member in the Computer Engineering Department, Baghdad University. She has some important published papers in international journals and conferences.

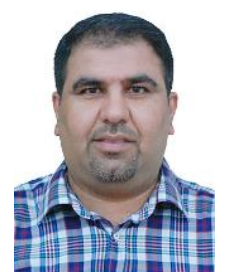

Dheyaa J. Kadhim received BSc in electrical engineering at Baghdad University; the MSc in electrical engineering at Baghdad University; the $\mathrm{PhD}$ in communication and information engineering at Huazhong University of Science and Technology in China. He is currently faculty member in the Electrical Engineering Department, Baghdad University, member of Iraqi Engineers Society, Editor and Reviewer at some International Science and Engineering Journals. Finally, he participated in many important conferences as a researcher, reviewer or as an editor such as WCNC,WiCOM, FCC, CMC, ICIS. 